\title{
Archéopages
}

Archéopages

Archéologie et société

45 | 2017

De la terre au pot

\section{Constantes et variations autour d'un modèle}

L'exemple des vases biconiques armoricains de l'âge du Bronze

Constants and variations around a model. The example of Armorican biconic vases from the Bronze Age

Constantes y variaciones alrededor de un modelo. El ejemplo de los jarros

bicónicos armoricanos de la Edad del Bronce

Julien Ripoche et Théophane Nicolas

\section{OpenEdition}

Journals

Édition électronique

URL : https://journals.openedition.org/archeopages/2481

DOI : 10.4000/archeopages. 2481

ISSN : 2269-9872

Éditeur

INRAP - Institut national de recherches archéologiques préventives

Édition imprimée

Date de publication : 1 mars 2018

Pagination : 6-15

ISSN : 1622-8545

Référence électronique

Julien Ripoche et Théophane Nicolas, "Constantes et variations autour d'un modèle », Archéopages

[En ligne], 45 | 2017, mis en ligne le 01 janvier 2020, consulté le 04 juin 2021. URL : http://

journals.openedition.org/archeopages/2481; DOI : https://doi.org/10.4000/archeopages.2481 


\section{Constantes et variations}

\section{autour d'un modèle}

\section{L'exemple des vases biconiques armoricains de l'âge du Bronze}

Julien Ripoche Universite Paris 1, UMR 8215, «Trajectoires "

Théophane Nicolas Inrap, UMR 8215, «Trajectoires»

1. Voir la rubrique

"Pratiques » dans

ce numéro.

2. Opération menée

de mars à juin 2010

sous la responsabilité

de Yoann Escats, Inrap.

3. J. Ripoche,

"Traditions céramiques

et dynamiques culturelles

à la transition des $\mathrm{III}^{\mathrm{e}}$

et $\mathrm{II}^{\mathrm{e}}$ millénaires en

Bretagne : Genèse

de l'âge du Bronze et interactions en Europe

atlantique (2300-1750 av.

J.-C.) », sous la direction

de F. Giligny, Université

Paris 1 , en cours.
Jusqu'à présent, les vases biconiques à carène haute et, plus largement, les assemblages céramiques de la culture des Tumulus armoricains étaient presque exclusivement attestés en contexte funéraire. Depuis les années 2000, avec l'essor de l'archéologie préventive, on constate un renouvellement important des données, notamment avec la mise au jour de contextes domestiques (Blanchet et al., à paraître). Ces découvertes permettent de disposer de contextes clos et bien datés, propices à la mise en place d'une approche technologique. Celle-ci est à même de renouveler les connaissances des productions céramiques en termes chronologique, typologique et fonctionnel ${ }^{\mathbf{1}}$. Si la mise en oeuvre d'une approche technologique du mobilier céramique du Bronze ancien atlantique est inédite, elle s'inscrit dans la continuité de celles développées depuis la fin des années 1990 pour le Néolithique (Giligny, 1993 ; Gomart, 2014 ; Colas et al., 2015) et d'autres aires chronoculturelles (Méry, 1991 ; Roux, 2003). Régionalement, seules les approches typologiques et typochronologiques ont été developpées jusqu'à présent (Le Carduner, 1993 ; Stévenin, 200o). Bénéficiant du renouvellement des données, l'approche technologique du mobilier céramique permet d'intégrer les nouveaux corpus domestiques à la réflexion générale d'un point de vue typochronologique, mais également sur le plan des dynamiques culturelles et des réseaux d'échanges entretenus à l'échelle de la façade atlantique, tout particulièrement au travers des phénomènes d'emprunts, de copies et d'imitations (Giligny, Mery, 2010).

\section{La restitution des chaînes opératoires}

Le mobilier céramique du site de Lannion «ZAC de Bel-Air » (Côtes-d'Armor) ${ }^{2}$ (Escats,
2013) a fait l'objet d'une étude technologique dans le cadre d'un travail doctoral ${ }^{3}$. La qualité de conservation de la céramique, son abondance et sa découverte au sein de contextes clos (en position de rejet dans les fossés) font de cet ensemble l'un des plus importants connus à ce jour pour l'âge du Bronze ancien de la façade atlantique (Nicolas T., 2013 ; Ripoche, 2014). Plus de 500 individus ont pu être pris en compte. Ils permettent une restitution au moins partielle des chaînes opératoires. Dans cet assemblage, on dénombre une trentaine de vases qui correspondent typologiquement aux formes biconiques à carène haute et ouverture resserrée. Au travers de la restitution de la chaîne opératoire de trois d'entre eux, nous illustrerons des savoirfaire à une échelle locale et envisagerons plusieurs pistes d'études pour une approche extra-régionale.

Le premier vase est de facture semi-grossière [ill. 1]. Le dégraissant est composé principalement d'inclusions de quartz, feldspath, mica et de schiste de taille nanométrique à millimétrique. La densité apparente est comprise entre 15 et $20 \%$. Ces caractères indiquent un approvisionnement local, probablement en périphérie directe du site. La cuisson apparaît peu homogène avec des teintes variant du beige au noir, liées vraisemblablement à une cuisson en meule ou en fosse. La partie inférieure du vase n'est pas conservée ; le fond et sa jonction avec la partie basse de la panse ne sont donc pas pris en compte. La partie basse de la panse est réalisée à l'aide de colombins (au minimum cinq) étirés et disposés en position oblique interne. Leur jonction est assurée par des pressions digitées discontinues encore visibles sur la partie interne du récipient. La jonction entre les parties basses et hautes de la panse s'opère 
à l'aide d'un colombin disposé en position oblique interne permettant plus aisément la réalisation d'une carène vive [ill. 1c]. La partie haute du vase est confectionnée à l'aide de deux colombins faiblement étirés disposés en position subhorizontale comme l'atteste l'épaisseur supérieure de la panse à ce niveau. En raison de la difficulté d'accès pour le potier à la partie interne de la panse haute, un procédé particulier est utilisé : il consiste en un fort étirement de la pâte depuis le bord vers la carène du récipient, laissant visibles de profondes marques verticales d'impressions digitées continues. L'excédent de pâte ramené au niveau de la carène est ensuite déplacé et étalé sur la partie basse de la panse à l'aide d'un outil de type lissoir [ill. 1b]. Par ce biais, le potier vise probablement à renforcer la carène. L'ordonnancement des macrotraces observables met en avant la grande régularité de la gestuelle du potier. L'orientation oblique des plages de lissage réalisées nous fait envisager l'usage d'un système rotatif de type tournette lors de la régularisation de la surface du récipient. Le bord est réalisé par la simple pose d'un colombin dépassant, à la fois à l'intérieur et à l'extérieur du vase, sur la partie sommitale de la panse [ill. 1d]. Le dernier colombin, peu étiré, est mis en forme sur la partie extérieure à l'aide d'un lissoir quand sa partie interne reste faiblement régularisée.

Une dichotomie forte des traitements de surface peut être observée entre l'aire externe et l'aire interne du vase, mais aussi entre les parties basse et haute de cette dernière. La surface externe du vase, traitée de manière homogène, révèle un lissage particulièrement soigné. Une trace d'outil conservée sur la surface extérieure basse de la panse est le seul argument en faveur de l'emploi d'un lissoir en lieu et place de l'usage de la technique de la main mouillée. Si les modifications de la surface externe ne permettent pas, a priori, d'exclure l'hypothèse d'un poli mal conservé, c'est davantage la réalisation ultérieure du décor sur une pâte encore humide qui plaide en faveur du lissage. A contrario, le lissage de la surface interne du vase oppose une partie basse traitée sommairement à la main mouillée et une partie supérieure sans traitement de surface particulier, laissant les stigmates des étapes précédentes et le dégraissant minéral bien visibles en surface. Seules de rares plages polies localisées sur le bord ont pu être identifiées. Elles sont imputables davantage à la mise en forme du bord qu'à son traitement final.

Le registre décoratif se décline en deux thèmes réalisés par incision à l'aide d'un outil pointu. Des chevrons ou hachures obliques alternées (selon la terminologie employée) sont réalisés depuis le bord vers la carène. En partie basse, c'est un enchaînement continu de triangles incisés, réalisés depuis la carène et orientés vers le bas de la panse, qui est mis en œuvre. Des lignes verticales parallèles au sein de chacun de ces motifs achèvent la création de ce second thème décoratif. De légers bourrelets visibles aux bords de ces incisions, comme l'absence de recouvrement du décor, plaident pour une réalisation de l'ensemble de ces ornements postérieurement à celle des traitements de surface [ill. 1a].

Le deuxième vase [ill. 2] est également de facture semi-grossière. Néanmoins, comme l'atteste la teinte rouge brique des parois en opposition avec un cœur sombre, la cuisson a été réalisée en atmosphère réductrice/oxydante. En dépit d'une fragmentation relativement importante du récipient, l'ensemble de la chaîne opératoire a pu être reconstitué. Le dégraissant de nature locale est de même type que celui du premier vase.

Le fond est réalisé à partir d'un colombin enroulé en spirale mis en forme par pressions digitées. La jonction avec la panse se fait depuis l'extérieur par étirement du premier colombin, de la panse sur la partie externe du fond, et par pressions digitées. Quatre colombins sont ensuite nécessaires pour former la partie basse de la panse. La carène est réalisée par l'ajout d'un colombin oblique interne puis un dernier lui est associé pour terminer la panse. Tous les colombins sont disposés en position oblique interne. On observe, sur un étirement de colombin conservé [ill. 2b], deux impressions unguéales qui caractérisent des incisions au doigt visant à améliorer la jonction et l'adhérence entre deux colombins. Les parois sont mises en forme par des pressions digitées discontinues encore observables sur la partie haute interne du récipient. Les colombins sont par ailleurs bien visibles en relief sur la paroi extérieure basse du récipient. Pour la réalisation du bord, un dernier colombin plus fin semble avoir été ajouté même si les tranches émoussées rendent difficile la validation de cette hypothèse. On peut, néanmoins, attester de sa mise en forme par étirement et/ou repli à la fois sur la paroi externe et sur la paroi interne du vase. Cette mise en forme permet d'élaborer un bord droit légèrement épaissi.

Des éléments participant à un système de préhension ou de suspension ont été placés au niveau de la carène. Ces languettes ont la particularité d'avoir une excroissance modelée à leur base. Cet élément vient s'enchasser dans une concavité aménagée dans la paroi au niveau de la carène formant un assemblage de type tenon-mortaise [ill. 2c]. Cet assemblage améliore l'adhérence de l'élément plastique à la paroi du vase et sa résistance aux contraintes.

Les surfaces externe et interne du fond sont grossièrement lissées. On observe sur la surface externe $\mathrm{du}$ fond de nombreuses plages oblongues relatives à l'emploi d'un lissoir de petite dimension [ill. 2a]. Si le même outil semble avoir été utilisé pour les finitions de l'ensemble de la paroi extérieure, il est en revanche difficile de se prononcer sur celui utilisé pour la surface interne. On observe néanmoins, comme pour le vase 
1. Observations de la chaîne opératoire de productions

céramiques de l'âge du

Bronze ancien armoricain.

Vase $n^{\circ} 1$, Lannion, Zac

de Bel-Air (Escats, 2013); vues de détail. a. Décor de triangle réalisé

en partie basse.

b. Mise en forme de la partie

interne haute.

c. Colombin en position

oblique interne pour la

réalisation de la carène.

d. Colombin en position

subhorizontale ajouté

pour le bord.
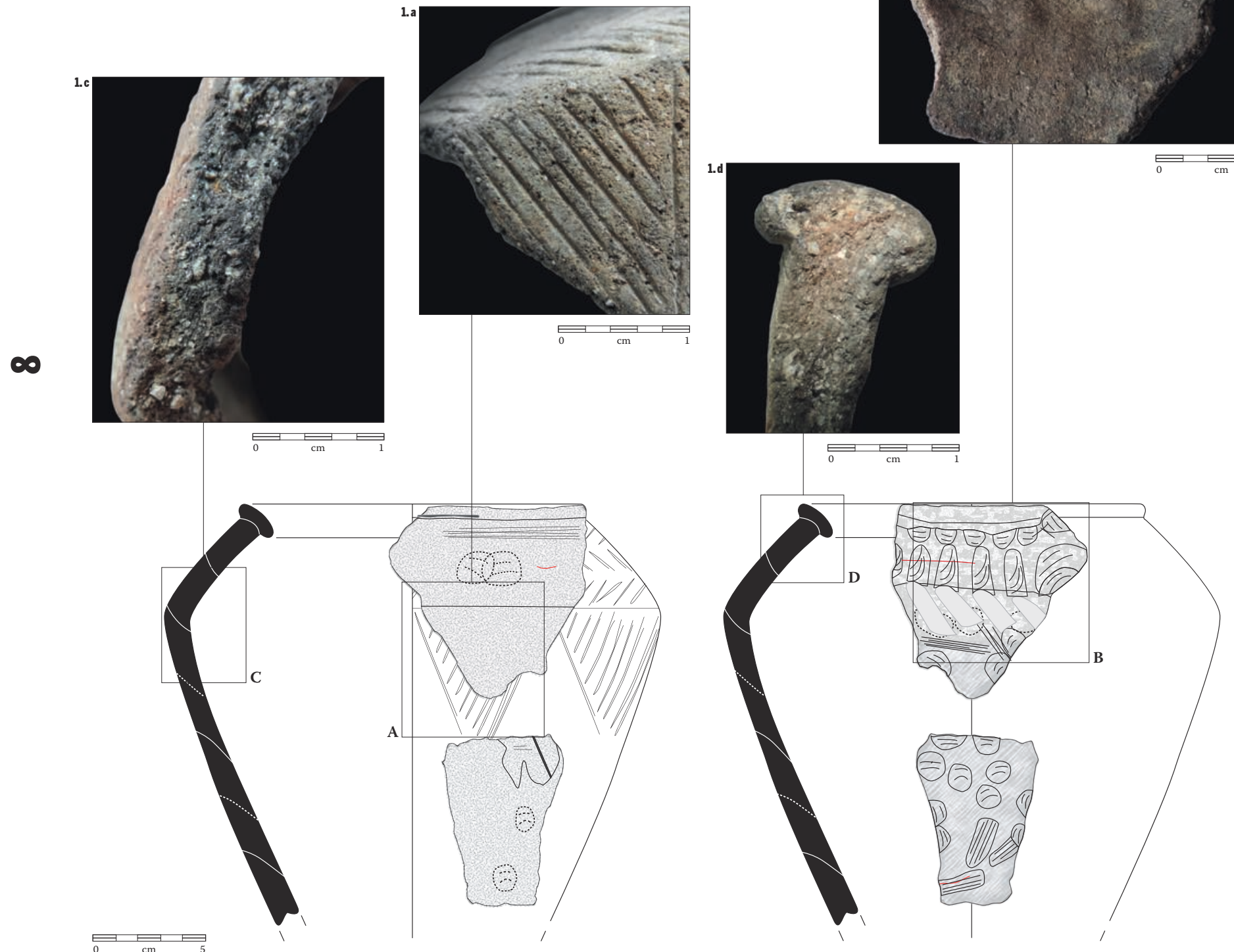

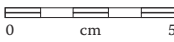

Non traité et/ou lissage peu investi

Lissage simple (plages de lissage visibles)

Lissage très investi et homogène (peu ou pas de plages de lissage visibles)

Brunissage (alternance de plages mates et polies)

Poli investi et homogène (peu ou pas de plages de polissage visibles)
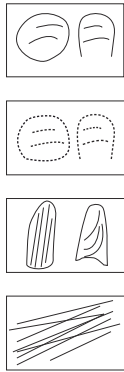

IV
Point de compression (impression digitée discontinue)

Point de compression supposé (impression digitée discontinue)

Plage de compression (impression digitée discontinue)

Stries fines (lissage type main mouillée)

Plage oblongue polie (polissage/ brunissage)

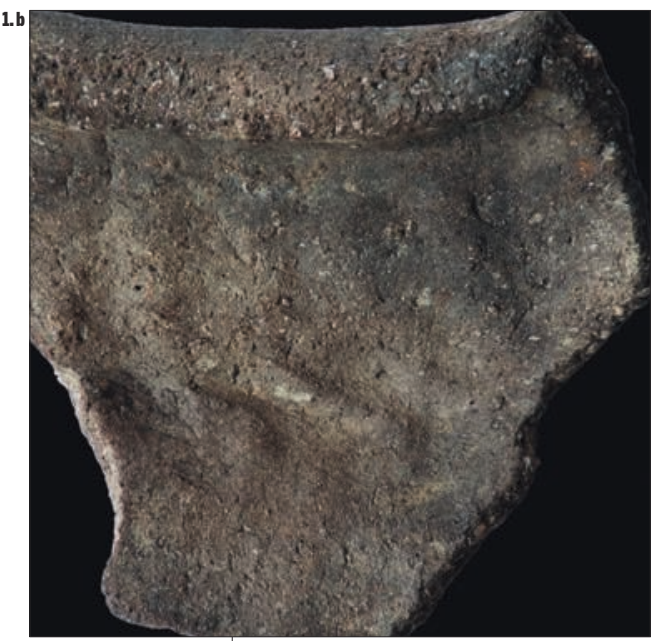

$\rightleftharpoons \stackrel{\mathrm{cm}}{\rightleftharpoons}$ 
de Bel-Air (Escats, 2013);

vues de détail.

a. Plages de lissage liées

à la régularisation du bord.

b. Coups d'ongle visant à

améliorer la jonction entre

deux colombins.

c. Aménagement d'une

concavité sur la paroi liée

à la pose d'une languette

avec tenon.

d. Réalisation du décor par coups d'ongle et étirement du bord sur la paroi externe du récipient.
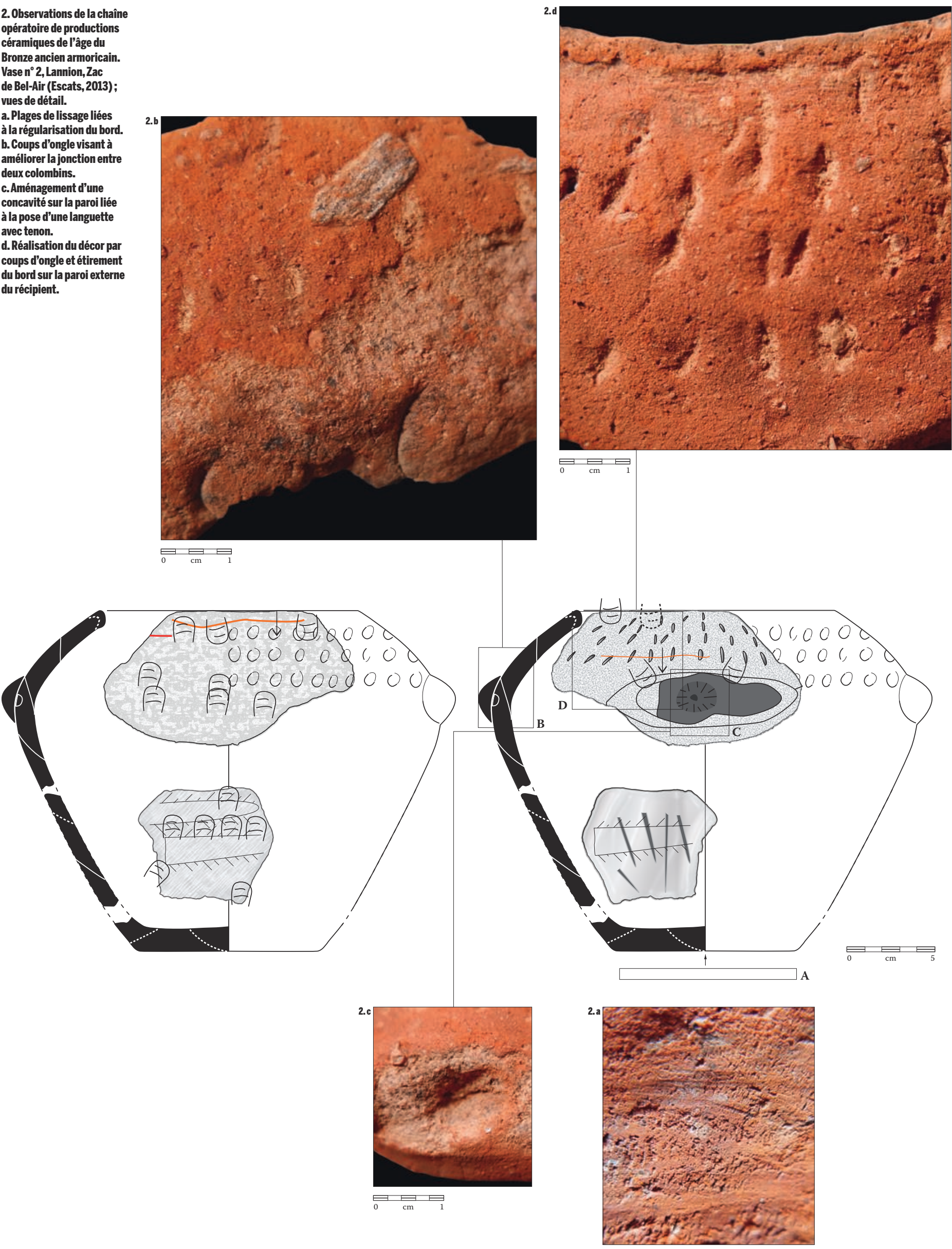
3. Observations de la chaîne

opératoire de productions

céramiques de l'âge du

Bronze ancien.

Vase ${ }^{\circ} 3$, Lannion, Zac de

Bel-Air (Escats, 2013);

vues de détail.
a.Arrachement partie

de la galette du fond.

b. Pose d'un colombin oblique

interne pour la réalisation

de la jonction entre le fond

et la panse.

c. Plages de polissage sur

la partie interne du vase,

sur une pâte à consistance

cuir, liées à la réalisation

d'un polissage simple.

d. Plages de polissage

visibles sur la partie externe

du vase liées à la réalisation

d'un polissage soigné

et homogène.
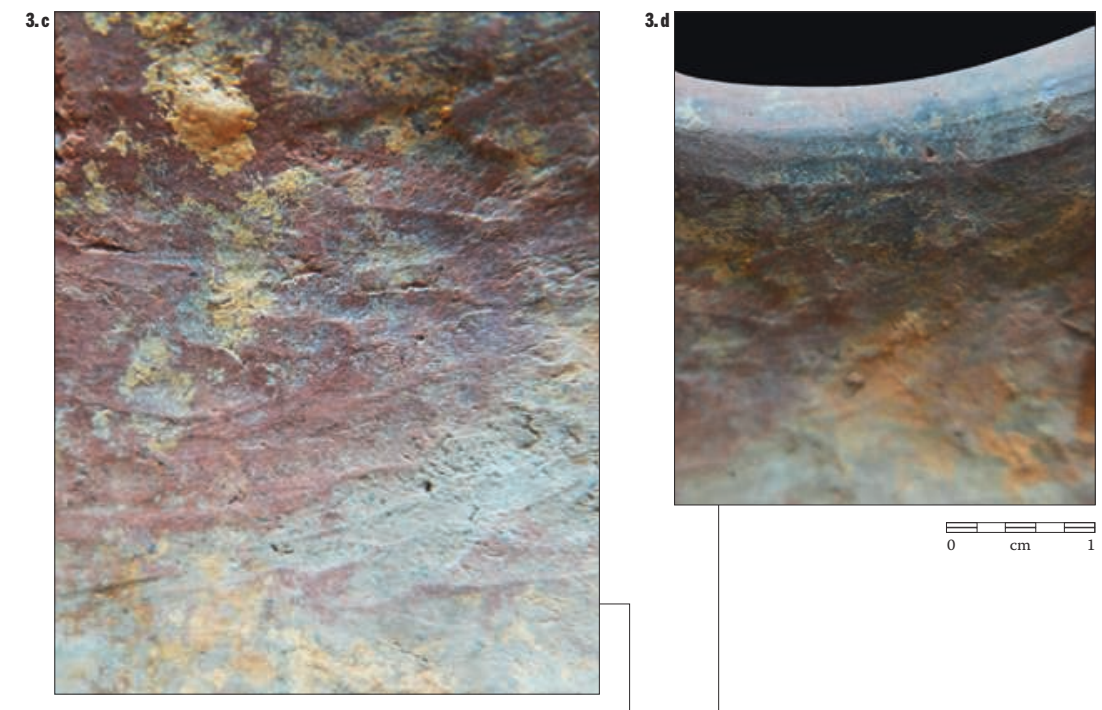

$\underset{\mathrm{cm}}{\rightleftharpoons} \rightleftharpoons$

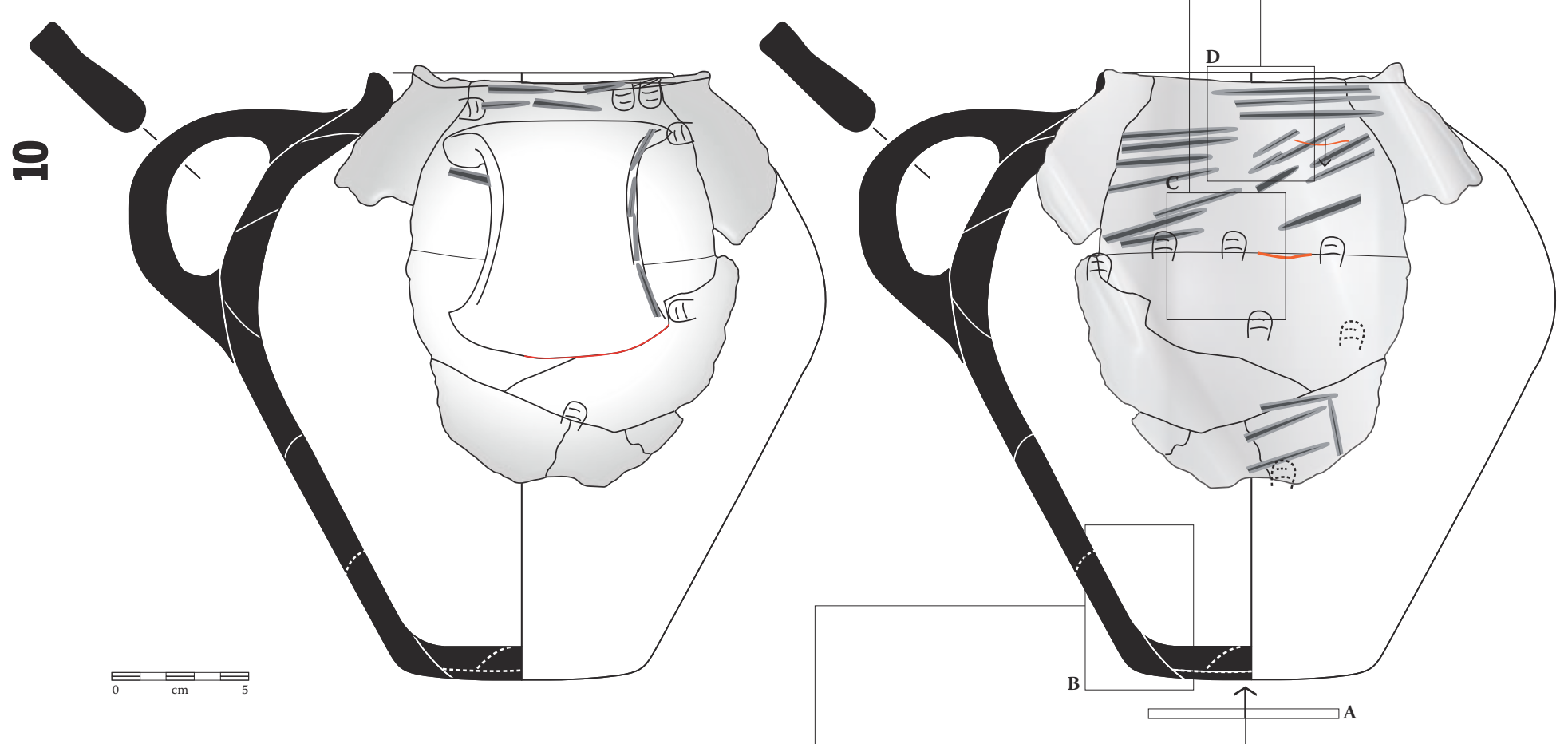

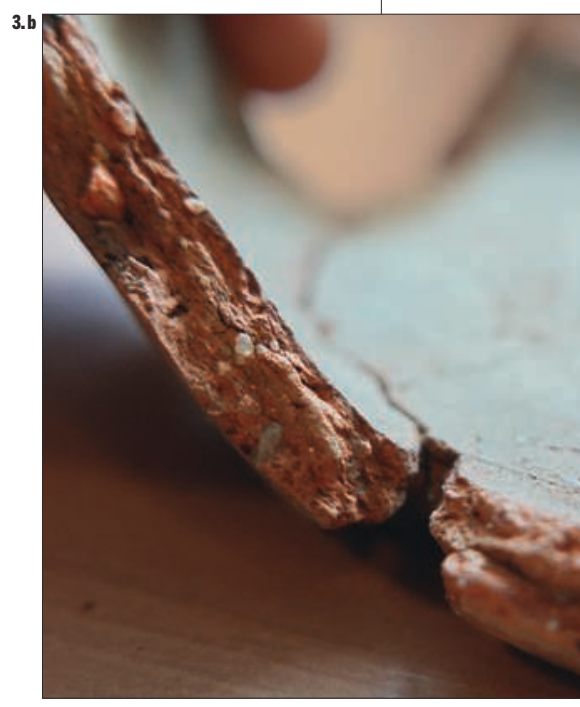

$\rightleftharpoons \underset{\mathrm{cm}}{\rightleftharpoons} \rightleftharpoons$

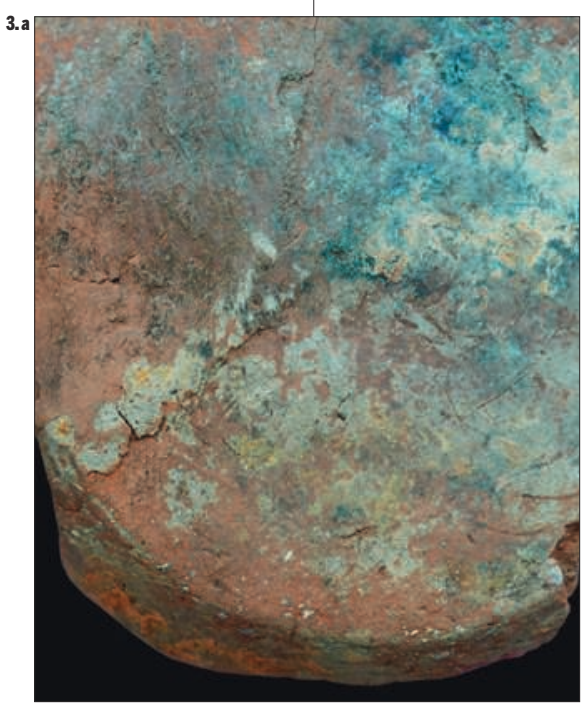

$\overline{\overline{\mathrm{cm}}} \stackrel{\overline{\mathrm{F}}}{\overline{\mathrm{F}}}$ 
précédent, une nette opposition entre la partie haute, très travaillée, et la partie basse du vase, à peine régularisée. Le bord, enfin, n'a pas fait l'objet de mise en forme ni de finition particulière : les étirements sur les parois externe et interne du vase sont encore bien visibles. En l'absence de recouvrement partiel lors du lissage, le décor semble par conséquent avoir été réalisé après les étapes de finitions. Il s'agit d'un décor linéaire d'impressions à l'ongle sur la partie haute de la panse, disposé de manière relativement anarchique et effectué sur une pâte encore humide [ill. 2d].

Le troisième vase [ill. 3] est un récipient de facture semi-grossière ; si la composition du dégraissant est comparable à celle des deux autres individus, la granulométrie est légèrement plus faible. La variété des couleurs de la matrice laisse supposer une cuisson hétérogène.

Des traces remarquables, localisées sur la surface externe du fond, indiquent qu'il a été façonné sur un support et que l'on s'est servi d'un outil pour le décoller de celui-ci. Les incisions subcirculaires visibles ainsi qu'un arrachement partiel du fond témoignent de la difficulté rencontrée par le potier lors de cette étape [ill. 3a]. Le fond en lui-même est élaboré à partir d'un colombin en spirale simple. Néanmoins, certains stigmates, comme l'arrachement partiel du fond, pourraient indiquer qu'une seconde galette a été ajoutée contre sa face extérieure pour le renforcer. La jonction entre le fond et la panse est simplement réalisée par étirement du premier colombin de la panse sur la paroi externe du fond et constitue un point de contrainte élevée [ill. 3b]. La partie basse de la panse est ensuite fabriquée à l'aide de colombins disposés cette fois en position oblique externe et peu étirés. Ils sont au nombre minimum de trois ; toutefois leur décompte exact demeure difficile à déterminer. Les jonctions de colombins sont assurées par des pressions digitées discontinues encore partiellement visibles sur la partie interne du vase en dépit des traitements de surface postérieurs. La jonction au niveau de la carène est, comme pour les exemples précédents, opérée à l'aide d'un colombin disposé en position oblique interne. La partie haute de la panse est confectionnée à l'aide de trois colombins peu étirés, disposés en position oblique interne. La jonction au niveau de la carène et entre ces colombins est assurée à l'aide d'impressions digitées discontinues. Le bord est réalisé par la mise en forme du dernier colombin de la partie haute de la panse, replié et étiré sur la paroi externe du vase.

L'anse est alors ajoutée au récipient préalablement aux traitements de surface. Elle est simplement accolée au niveau de la carène et sous le bord, puis ses extrémités sont étirées sur la surface de la panse afin d'en optimiser l'attache. D'importantes fissures sont visibles à la jonction de l'anse et de la panse, ce qui indique un point de faiblesse à ce niveau. Le traitement de surface de la partie externe du fond est caractérisé par un lissage (probablement à la main mouillée). On notera l'absence de reprise de la surface extérieure du fond présentant un arraché de la galette d'argile. La surface interne est, elle aussi, lissée selon la même technique. La surface interne de la partie basse de la panse a donné lieu à un lissage simple en deux étapes : un premier traitement peu soigné à l'aide d'un lissoir visant à régulariser grossièrement la surface, puis un second à la main mouillée pour donner à la paroi son aspect définitif. Le traitement de la partie haute est néanmoins réalisé au lissoir en raison de l'état de séchage de la pâte et les traces visibles caractérisent davantage un brunissage qu'un lissage en raison de la présence de quelques plages polies en alternance avec des plages mates [ill. 3c].

Malgré l'altération relative de la surface extérieure de la panse, un nombre important de stigmates ont ainsi pu être reconnus : un polissage simple a été effectué à l'aide d'un outil de type galet. Ce traitement apparaît bien homogène, y compris sur les parties interne et externe de l'anse. La surface du bord a été traitée de manière comparable [ill. 3d].

\section{Des productions standardisées?}

En dépit d'une certaine variabilité des chaînes opératoires, un certain nombre de points communs plus ou moins discriminants ont été identifiés et sont généralisables au Bronze ancien armoricain et à sa périphérie. Pour l'élaboration des fonds, on observe le recours à l'utilisation d'un colombin en spirale mis en forme par pressions digitées, sans autre aménagement particulier, associé à une jonction depuis l'extérieur avec la panse. Cette technique largement majoritaire au sein de ce corpus est aussi observée de manière récurrente sur d'autres sites de Bretagne comme Plouisy, «Zac de Kérisac » (Côtes-d'Armor) (Mentele, 2013), ou encore Pleurtuit « Cap Emeraude-secteur $2 »$ (Ille-et-Vilaine) (Hamon, 2014) ; elle semble caractéristique du Bronze ancien armoricain (Ripoche, 2016).

La panse est façonnée à partir de colombins semi-épais (diamètre supérieur à $2 \mathrm{~cm}$ ), étirés et disposés le plus souvent en position oblique interne et ponctuellement en position oblique externe dans le cas du vase $n^{\circ} 3$. La jonction au niveau de la carène est toujours assurée par l'ajout d'un colombin particulièrement étiré en position oblique interne, permettant la réalisation d'une forte angulation. Dans les trois cas présentés, deux ou trois colombins supplémentaires seulement sont nécessaires à la mise en œuvre de la partie haute du vase. Les bords renvoient néanmoins à deux techniques distinctes, à savoir l'ajout d'un dernier colombin terminal pour réaliser un bord surépaissi ou bien la simple mise en forme du dernier colombin de la panse pour créer un bord sortant. 


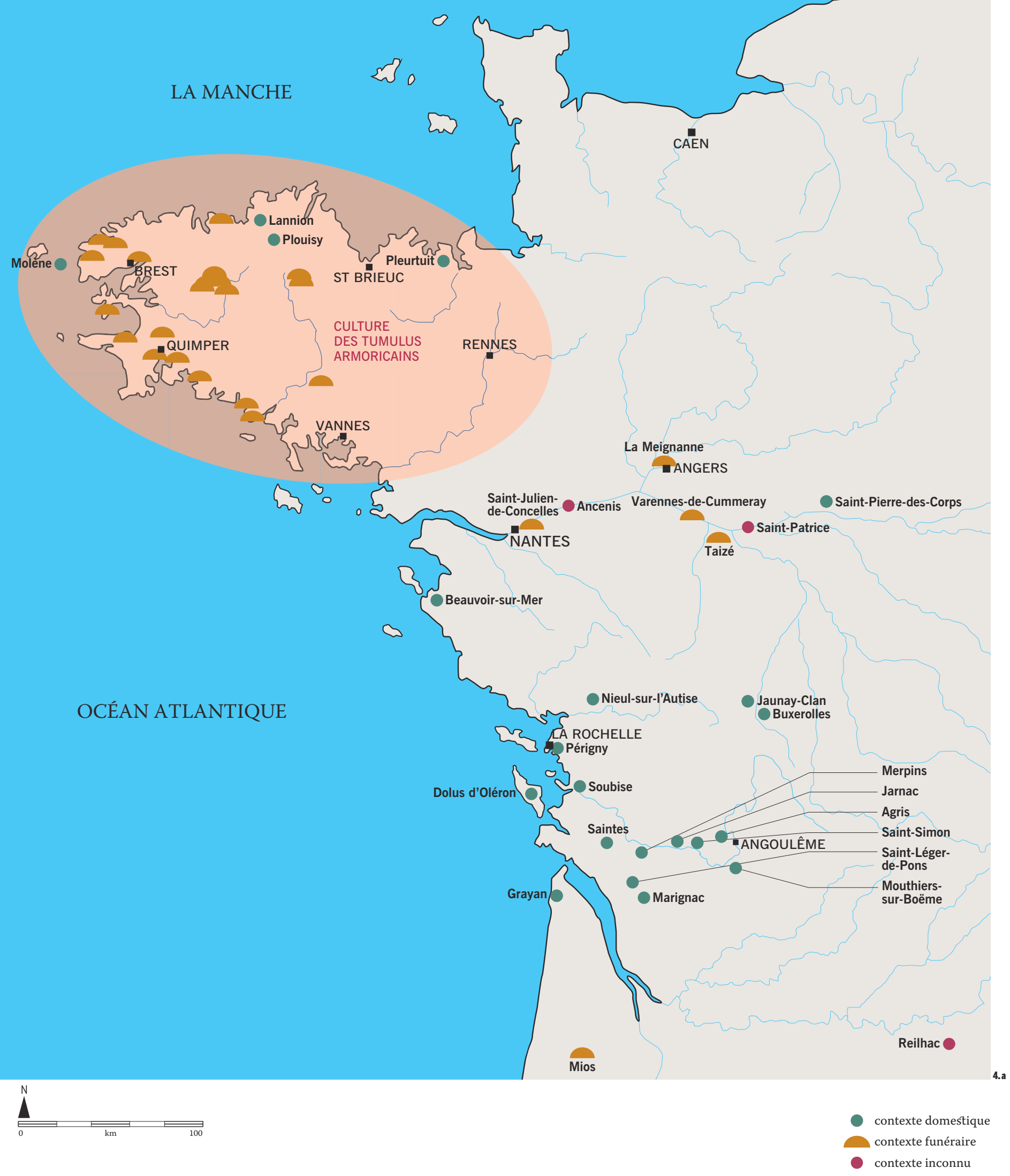

4.a. Répartition spatiale des principaux sites ayant livré des céramiques biconiques présentant des ressemblances avec les productions armoricaines (Nicolas T.,, 2013, complété). 

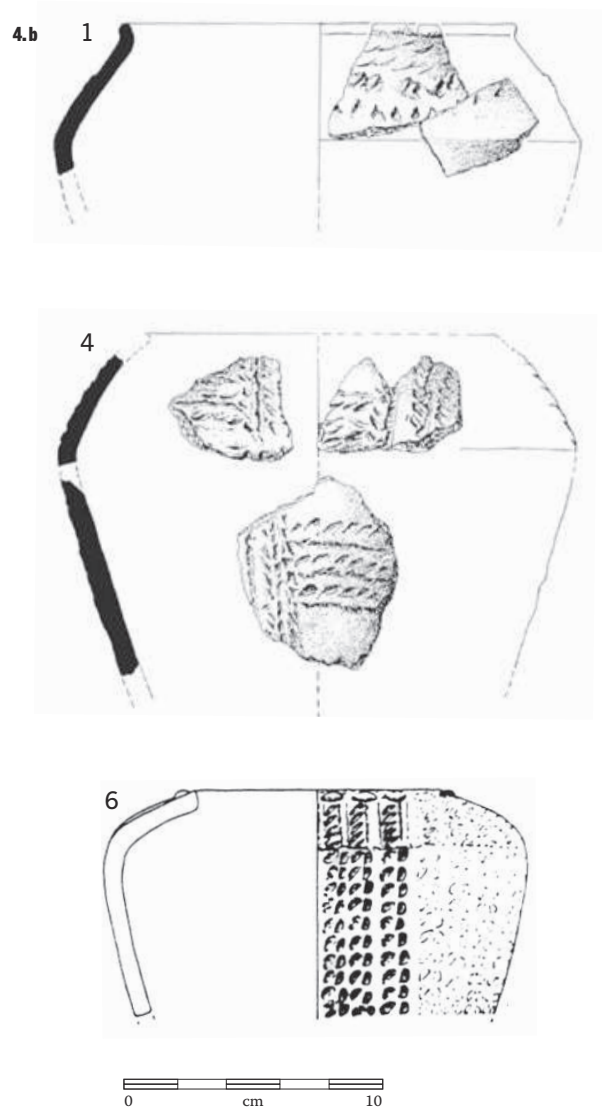
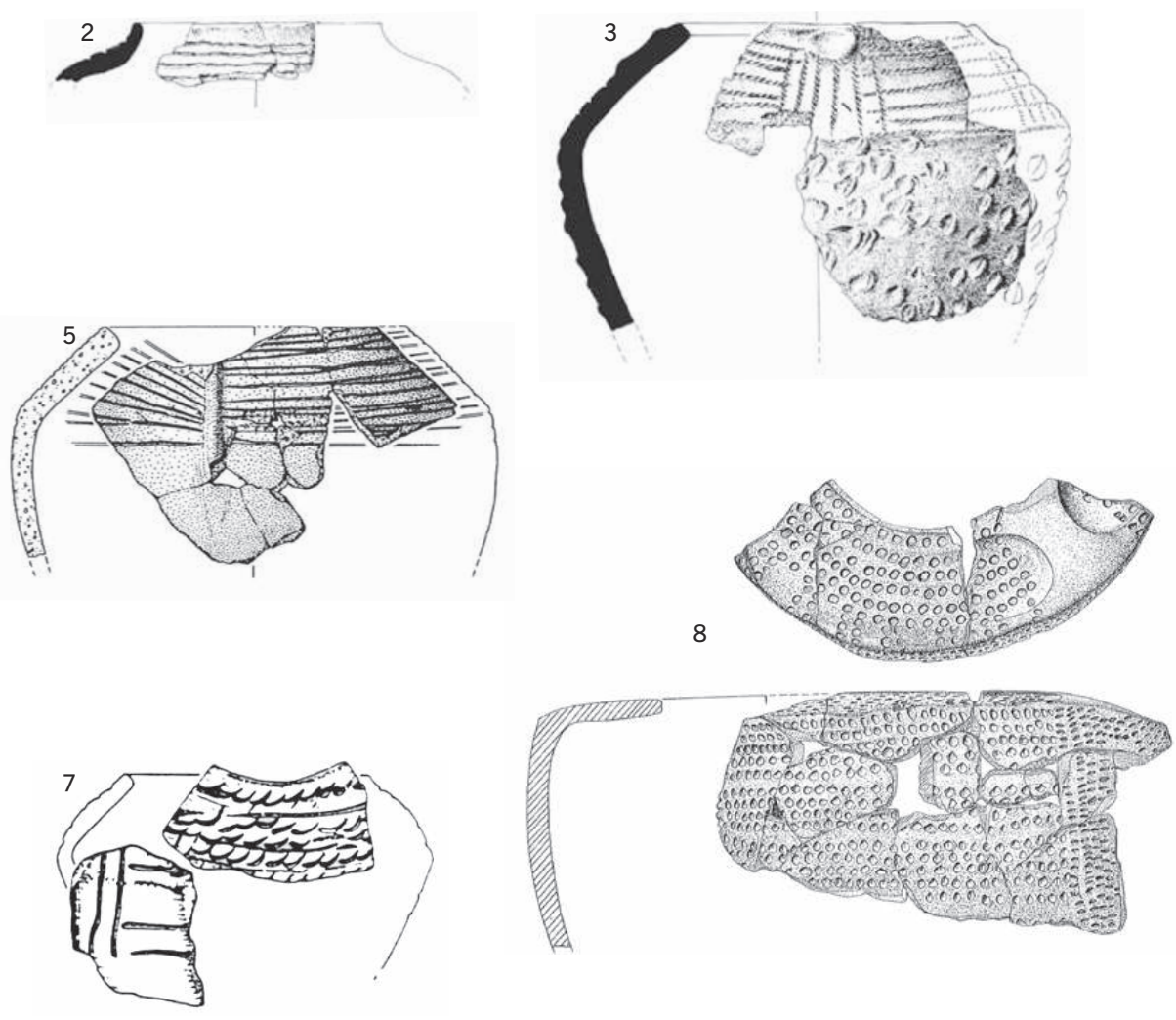

$m$

La fabrication par le potier de systèmes de préhension pour ces productions est elle aussi fréquente, néanmoins leurs mises en œuvre renvoient à des savoir-faire distincts : simple application sur la paroi, système de tenon et mortaise, etc. On observe aussi, par exemple, le peu de soin apporté à la régularisation de la partie haute interne qui découle de la volonté affichée des potiers de s'investir en priorité sur les parties visibles du vase. Cette dernière observation nous amène à envisager l'élaboration de ces vases d'un seul tenant et non à partir de deux parties distinctes : en effet cette technique aurait permis une régularisation plus aisée de la partie haute et au contraire une concentration de stigmates visibles au niveau de la carène (ce qui ne pourrait être éventuellement envisagé ici que pour le vase $n^{\circ} 1$ ).

S'il semble évident, au vu des différences constatées, que ces vases sont l'œuvre de trois potiers différents (différence dans les techniques de finition, d'ornementation, de mise en forme et de régularisation du bord, etc.), les procédés communs reconnus dans l'élaboration de ces trois vases nous permettent de définir un fond commun, synonyme d'une tradition technique armoricaine dans l'élaboration des vases biconiques à carène haute de l'âge du Bronze ancien. La fabrication d'un vase est guidée par trois facteurs principaux : le standard (qui dépend lui-même de facteurs culturels), les techniques mises en œuvre (la technologie, savant mélange d'héritages, d'échanges et d'innovations) et, bien entendu, des spécificités techniques propres à la «main » du potier. Dès lors, les enseignements à tirer de ces observations sont de plusieurs niveaux. Ils permettent ainsi de s'intéresser, par le biais des techniques héritées, à la relation complexe entretenue entre les différentes productions couvrant la transition du $\mathrm{III}^{\mathrm{e}}$ millénaire et aussi de reconsidérer la place occupée par le domaine armoricain dans les nouveaux réseaux d'échanges, à l'aube de l'âge du Bronze, sur la façade atlantique. Enfin, cette démarche permettra de s'interroger sur les évolutions propres au Bronze ancien, de reconsidérer la place de ces productions dans la chronologie de cette période.

\section{Des productions destinées à un usage funéraire?}

L'opposition entre les traitements des vases $n^{\circ} 1$ et 2 [ill. 1 et 2] et du vase $n^{\circ} 3$ [ill. 3] est intéressante si l'on tient compte des contextes dont ils sont issus. En effet, les deux premiers, qui proviennent 
du fossé de l'enceinte, sont interprétés comme du mobilier domestique. A contrario, le vase $\mathrm{n}^{\circ} 3$, issu du tumulus contigu à l'enceinte, se caractérise par un travail de finition particulièrement prononcé, en contraste avec un façonnage et une mise en forme plus hasardeuse. Le poli de surface particulièrement bien réalisé sur le vase funéraire le distingue de la production domestique de l'enceinte pour laquelle le lissage est largement privilégié et où de rares cas de polissage sont attestés, seulement pour des vases en céramique fine de type gobelet.

Néanmoins, un certain nombre de défauts liés à la fabrication ont été identifiés et contrastent avec ce traitement de surface soigné : convexité du fond liée à l'arrachement d'une partie de celui-ci lors de sa désolidarisation avec le support sur lequel s'est opéré son façonnage, dissymétrie de la forme, tout comme l'absence de renfort au niveau de l'anse. Tous ces éléments portent à croire que ce vase n'aurait pas été, a priori, fonctionnel pour un usage au sein d'un vaisselier domestique.

Cette observation corrobore une constatation réalisée dans d'autres contextes chronoculturels sur l'existence de productions à vocation funéraire : certaines montrent des finitions très travaillées, mais souffrent d'une qualité de façonnage inférieure à celle observée sur les productions domestiques ; pour d'autres, le potier a respecté les canons généraux du profil en usage, mais s'est exempté des opérations de finition ou de décoration. Ces différentes observations posent la question de productions exclusivement destinées au rituel funéraire, où la gestuelle du dépôt est en lien avec la valeur symbolique du vase ou bien en relation avec ce qu'il pouvait contenir.

\section{D’intenses réseaux d'échanges atlantiques?}

À l'âge du Bronze ancien, l'aire de répartition des formes biconiques à carène haute s'étend le long de la façade atlantique depuis, a minima, le Centre-Ouest de la France jusqu'au sud des îles Britanniques. Néanmoins, localement, les registres décoratifs diffèrent et des convergences de formes peuvent être observées à une échelle locale. Au sein de cette aire, le Centre-Ouest de la France concentre un nombre important de vases de type armoricain qui ont été mis au jour pour l'essentiel au sein d'ensembles domestiques [ill. 4a]. Plusieurs de ces récipients correspondent morphologiquement au type « biconique à carène haute », mais plusieurs différences apparaissent par rapport aux canons des types armoricains, du point de vue des décors notamment. Ainsi, les thèmes de triangles incisés ou de chevrons, récurrents en Bretagne, laissent ici place à des décors linéaires imprimés (à la cordelette réelle ou imitée), compartimentés au sein de «métopes » par des motifs verticaux, qui définissent le canon régional. Ce thème, toujours appliqué en partie haute du vase, est le plus souvent associé avec des décors simples et linéaires réalisés à l’ongle en partie basse sur tout le pourtour du vase [ill. 4b].

Du point de vue technologique, on note également une certaine variété, qui permet d'exclure que ces récipients du Centre-Ouest de la France soient exogènes, en provenance d'Armorique au sens large. Les vases de JaunayClan « la Viaube 1 » (Lavoix, 2013, vol. 2, pl. 22, $\mathrm{n}^{\circ} 2$ ) et de Jarnac « les Champs Battazards » (Ranché et al., 2007, fig. 5) illustrent ce constat. Pour le premier de ces vases, la jonction au niveau de la carène n'est pas réalisée par la pose d'un colombin depuis l'intérieur, mais par un véritable colombin charnière peu étiré qui assure la jonction entre la partie haute et la partie basse du vase. Deux colombins peu étirés sont ensuite posés pour la fabrication de la panse haute, le dernier faisant office de « bord droit » sans reprise ou ajout de matière particulier [ill. $4 b, n^{\circ}$ 1]. Pour le second vase, l'ensemble de la chaîne opératoire peut être reconstitué. On observe plusieurs différences majeures : le fond est élaboré à partir d'une simple galette d'argile associée à un colombin périphérique qui joue le rôle d'articulation de la jonction de la panse et du fond. Les colombins de la partie basse de la panse, plus petits, sont faiblement étirés et présentent des jointures subhorizontales au nombre de sept ou huit. La jonction au niveau de la carène est effectuée depuis l'extérieur comme le montre la fracture présente sur tout le pourtour du vase à ce niveau. Trois colombins en position oblique externe permettent ensuite la réalisation de la partie haute de la panse avant qu'un dernier colombin, plus fin, soit ajouté et replié pour la mise en forme du bord [ill. 4b, n²].

Pour ces deux cas, il reste délicat d'établir s'il s'agit d'une imitation des modèles armoricains sur le plan strictement typologique, mais la technologie mise en œuvre, la réalisation du vase lui-même et de ses décors permettent facilement de les distinguer de ces modèles. La présence sur le second vase d'anses davantage décoratives que fonctionnelles vient renforcer ce constat, transformant un élément fonctionnel dans le domaine armoricain en une évocation dont la présence peut marquer une certaine charge symbolique. Ce constat est renforcé par la présence au sein du corpus du Centre-Ouest de plusieurs autres occurrences de même type [ill. $4 b, n^{\circ} 3$ et 4 ].

Hormis de rares cas, la répartition de ces productions en dehors du domaine armoricain se limite à des sites proches du domaine maritime ou établis à proximité de réseaux fluviaux d'importance (la Loire et la Charente notamment) (Nicolas T., 2013, fig. 295). Les transferts déjà observés du point de vue typologique se confirment et s'étoffent davantage à l'échelle technologique. Ces données nouvelles permettent ainsi d'appréhender le dynamisme des échanges entretenus le long de la façade atlantique et 
